\title{
Unsupervised Learning of Brain States from fMRI Data
}

\author{
F. Janoos ${ }^{1,2}$, R. Machiraju ${ }^{1,2}$, S. Sammet ${ }^{2}$, M.V. Knopp ${ }^{1,2}$, and I.Á. Mórocz ${ }^{3}$ \\ ${ }^{1}$ Dept. of Computer Science, The Ohio State University, USA \\ 2 Dept. of Radiology, The Ohio State University, USA \\ 3 Dept. of Radiology, Harvard Medical School, USA
}

\begin{abstract}
The use of multivariate pattern recognition for the analysis of neural representations encoded in fMRI data has become a significant research topic, with wide applications in neuroscience and psychology. A popular approach is to learn a mapping from the data to the observed behavior. However, identifying the instantaneous cognitive state without reference to external conditions is a relatively unexplored problem and could provide important insights into mental processes. In this paper, we present preliminary but promising results from the application of an unsupervised learning technique to identify distinct brain states. The temporal ordering of the states were seen to be synchronized with the experimental conditions, while the spatial distribution of activity in a state conformed with the expected functional recruitment.
\end{abstract}

\section{Introduction}

Decoding the cognitive state of a subject from neuroimaging data has always been an important problem in cognitive neuroscience. Recently, this topic has gained prominence with the use of multi-variate pattern recognition (MVPR) to learn complex patterns in fMRI data[1]. Their multivariate nature makes them sensitive to patterns in groups of voxels that individually do not show much structure but as a whole contribute to mentation. Such techniques has been applied to the study of visual[1] and auditory perception, motor tasks[2], word recognition[3], lie-detection, etc., and may have applications in cognitive neuroscience [4], brain-machine interfaces [5], real-time biofeedback [2], etc. The reader is referred to [1] for a plenary review of this topic.

Typically, MVPR in fMRI tries to learn a mapping between the data and labels describing the prevailing attribute of interest (viz. presented stimuli and subject responses) thus only explaining observable attributes of mental processes. However, the identification of covert cognitive states which do not directly relate to experimental conditions is a relatively unexplored problem and could provide important insights into a person's subjective experience from a non-invasive measurement of brain activity. In contrast, sequences of "microstates" have been observed in EEG recordings, ranging from $70 \mathrm{~ms}$ to $150 \mathrm{~ms}$ and corresponding to a typical quasi-stable topography of electric field potentials in the brain[6]. They are believed to reflect the activation of different neurocognitive networks, and may be the "atoms of thought" that constitute the seemingly continual "stream of consciousness". While, temporal limitations would prevent access to these faster phenomena, fMRI is suited to reveal relatively longer lasting cognitive phenomena, such as attention, intention, planning, working memory, decision making, etc., that do not necessarily correspond to observable attributes. 
In this paper, we present a method to learn distinct states of activity distribution from fMRI data in an unsupervised fashion. The Earth Mover's Distance (EMD)[7] is used measure the difference between the distributions of activity in the cerebral cortex of two fMRI scans, and computed using a fast approximation method based on recursive aggregation. The EMD is used to derive a diffusion distance, which is aware of the geometry of the underlying low-dimensional manifold spanned by the data. The scans are then grouped using agglomerative hierarchical clustering in this low dimensional space, where each cluster represents a characteristic distribution of activity in the brain. The method was tested on an fMRI study of arithmetical abilities. Clusters corresponding to distinct mental states were identified. The temporal transitions between the states were observed to be synchronized with the experimental conditions, even though no information about the experiment was used. The observed brain activity in each state was found to agree with the expected neural recruitment during the corresponding mental task, thus reaffirming the ability of fMRI to study cognitive states of the brain.

The layout of the rest of the paper is as follows: In Sect. 2, details about the distance metric and clustering are presented, followed by a discussion of the results in Sect. 3 . Finally, we conclude with some remarks and outline future directions in Sect. 4.

\section{Method}

Preprocessing: The acquired data are subjected to routine pre-processing in SPM8 [8] (viz. bias-field correction, normalization to an atlas space, segmentation, motion correction and co-registration). The fMRI data are then de-noised using a wavelet-based Wiener filter[9], and further motion correction is performed using spatial ICA [10]. The mean volume of the time-series is then subtracted from each volume, and the white matter is masked out. All further processing is done in the grey matter to reduce the dimensionality of the data and to eliminate confounding influences from the white matter, which theoretically should not exhibit a BOLD effect. Each subject is processed independently.

Earth Mover's Distance: To group the fMRI scans $S(t), t=1 \ldots T$ into clusters of characteristic activity signatures, we need a measure of the difference between two scans. The Earth Mover's Distance[7] is well-known metric to compare two signatures $P$ and $Q$ defined over an arbitrary domain $\mathbb{X}$, given the ability to measure distances $d(x, y), \forall x, y \in \mathbb{X}$. It is defined in terms of an optimal flow $f^{*}: \mathbb{X} \times \mathbb{X} \rightarrow \mathbb{R}$, that minimizes the transportation problem $f^{*}=\arg \min _{f} \sum_{x, y \in \mathbb{X}} f(x, y) d(x, y)$, subject to the constraints: (i) $f(x, y) \geq 0$; (ii) $\sum_{y} f(x, y) \leq P(x)$; (iii) $\sum_{x} f(x, y) \leq Q(y)$; (iv) $\sum_{x, y} f(x, y)=\min \left\{\sum_{x} P(x), \sum_{y} Q(y)\right\}$, where $x, y \in \mathbb{X}$. The Earth Mover's distance is then $\operatorname{EMD}(P, Q)=\left(\sum_{x} \sum_{y} f^{*}(x, y) d(x, y)\right) /\left(\sum_{x} \sum_{y} f^{*}(x, y)\right)$. Since the domain $\mathbb{X}$ is the set of voxels in the grey matter, the geodesic distance $d(x, y)$ between two voxels $x, y \in \mathbb{X}$ is used. This is defined as the shortest path length on a graph $\mathbb{G}(\mathbb{X}, \mathbb{E})$, with grey matter voxels as nodes, and $(x, y) \in \mathbb{E}$ if and only if $x$ and $y$ are spatially adjacent to each other. Edge weights are $w(x, y)=\|x-y\|_{2}$, their Euclidean distance in physical space.

One major strength of the EMD over voxel-wise metrics, is that it allows for partial matches, and small differences between the signatures will result in a small EMD[7]. 
While there exist efficient algorithms for computing the EMD based on the transportation problem, it exhibits a worst-case complexity of $\mathcal{O}\left(|\mathbb{X}|^{3} \log |\mathbb{X}|\right)$. For an fMRI study with voxel size of $3 \times 3 \times 3 \mathrm{~mm}^{3}$, the number of grey matter voxels is $\approx 5 \times 10^{4}$, giving a running time of $\mathcal{O}\left(10^{14}\right)$. If the number of scans is $T$, it will require $T(T-1) / 4$ number of comparisons, making the pair-wise EMD computation prohibitively expensive. However, since $d(x, y)$ is now a shortest-path distance, not an Euclidean distance, standard approximations to the EMD are not applicable.

EMD Approximation: The problem is made tractable using a recursive approximation as follows: Let $S_{1}$ and $S_{2}$ be two scans with $|\mathbb{X}|$ voxels in the grey matter. Starting with $j=J>1$ create a low resolution graph $\mathbb{G}_{j}$ of $|\mathbb{X}| / 2^{j}$ nodes. Each node represents a set $\mathbb{I}_{n}^{j}, n=1 \ldots|\mathbb{X}| / 2^{j}$ of $2^{j}$ voxels, grouped based on their spatial proximity. The EMD is computed between $S_{1}$ and $S_{2}$ on this low-res graph as $E M D_{j}\left(S_{1}, S_{2}\right)$. If the edge weights and vertex values of $\mathbb{G}_{j}$ are calculated appropriately, then $\operatorname{EMD}\left(S_{1}, S_{2}\right)$ $\geq E M D_{j}\left(S_{1}, S_{2}\right)$, i.e. it is a lower bound on the EMD on the original mesh. If $E M D_{j}\left(S_{1}, S_{2}\right) \geq \tau_{j}$, where $\tau_{j}$ is a threshold, then we approximate $\operatorname{EMD}\left(S_{1}, S_{2}\right)$ by $E M D_{j}\left(S_{1}, S_{2}\right)$, otherwise set $j=j-1$ and repeat. Continue until $j=0$, in which case $E M D\left(S_{1}, S_{2}\right)=E M D_{0}\left(S_{1}, S_{2}\right)$.

Next, we explain how to correctly aggregate a set of vertices $\mathbb{I} \subseteq \mathbb{X}$ into a new vertex $x^{\prime}$, so that the EMD computed on this reduced graph $\mathbb{G}^{\prime}\left(\mathbb{X}^{\prime}, \mathbb{E}^{\prime}\right)$ is less than that on the original graph $\mathbb{G}(\mathbb{X}, \mathbb{E})$. For explicatory purposes, let $\sum_{x} S_{1}(x)=\sum_{x} S_{2}(x)$, though the reasoning holds for the general case. Let $S=S_{2}-S_{1}$ be the difference between the two distributions. Then, the optimal flow $f^{*}$ from $S_{1}$ to $S_{2}$ on $\mathbb{G}$ is the solution to the transportation problem, subject to the constraints $\sum_{y} f(y, x)-\sum_{y} f(x, y)=$ $S(x)$ and $f(x, y) \geq 0$, for all $x, y \in \mathbb{X}$. Now, the total cost can be partitioned as $\sum_{x, y \in \mathbb{X}} f^{*}(x, y) d(x, y)=\sum_{x, y \in \mathbb{I}} f^{*}(x, y) d(x, y)+\sum_{x, y \in \mathbb{X}-\mathbb{I}} f^{*}(x, y) d(x, y)+$
$\sum_{x \in \mathbb{I}, y \in \mathbb{X}-\mathbb{I}} f^{*}(x, y) d(x, y)$.

Firstly, through a conservation of mass argument, we see that the total flow from $x \in$ $\mathbb{I}$ to all $y \in \mathbb{X}-\mathbb{I}$ must be $\sum_{x \in \mathbb{I}, y \in \mathbb{X}-\mathbb{I}} f(x, y)=\sum_{x \in \mathbb{I}} S(x)$. Let $f^{\dagger}$ be the optimal solution to the transportation problem on the graph $\mathbb{G}^{\prime}$ where the value $S\left(x^{\prime}\right)=\sum_{x \in \mathbb{I}} S(x)$. Also, let $w^{\prime}\left(x^{\prime}, y\right)=\min _{x \in \mathbb{I}} w(x, y)$, and $d^{\prime}$ be the shortest path lengths on $\mathbb{G}^{\prime}$. Therefore, the last term $\sum_{x \in \mathbb{I}, y \in \mathbb{X}-\mathbb{I}} f^{*}(x, y) d(x, y) \geq \sum_{y \in \mathbb{X}^{\prime}} f^{\dagger}\left(x^{\prime}, y\right) d^{\prime}\left(x^{\prime}, y\right)$. Secondly, it can be proved though contradiction that the first term $\sum_{x, y \in \mathbb{I}} f^{*}(x, y) d(x, y) \geq$ $\sum_{x, y \in \mathbb{I}} f^{\circ}(x, y) d(x, y)$, where $f^{\circ}$ is the solution to the transportation problem restricted to the subgraph $\mathbb{I}$, subject to the constraints: (i) $f(x, y) \geq 0$; (ii) $\sum_{y} f(x, y) \leq S_{1}(x)$; (iii) $\sum_{x} f(x, y) \leq S_{2}(y)$; (iv) $\sum_{x, y} f(x, y)=\min \left\{\sum_{x} S_{1}(x), \sum_{y} S_{2}(y)\right\}-\sum_{x} S(x)$, with $x, y \in \mathbb{I}$.

This subproblem could again be solved using the above recursive approximation scheme. However, if $|\mathbb{I}|$ is small enough so that the geodesic and $\ell_{2}$ distances between its vertices are approximately equal, then the approximation $\operatorname{EMD}(P, Q) \geq\|\bar{P}-\bar{Q}\|_{2}$ can be used, where $\bar{P}$ and $\bar{Q}$ are the centroids of the signatures $P$ and $Q$ respectively[7].

Therefore, through the recursive approximation scheme we get a lower bound as per $E M D\left(S_{1}, S_{2}\right) \geq E M D_{j}\left(S_{1}, S_{2}\right)+\sum_{n=1}^{|\mathbb{X}| / 2^{j}} E M D\left[\mathbb{I}_{n}^{j}\right]\left(S_{1}, S_{2}\right)$, where $E M D\left[\mathbb{I}_{n}^{j}\right]$ $\left(S_{1}, S_{2}\right)$ is the EMD restricted to the subgraph defined by $\mathbb{I}_{n}^{j}$. 
Diffusion Distance: While the EMD provides a good comparison of scans with similar activity patterns (i.e. low EMD), its suitability to quantify the distance between scans with larger differences is more uncertain, apart from the fact that for such comparisons, we have only an approximate EMD. Then, assuming the accuracy of the EMD only in local neighborhoods on the manifold spanned by BOLD images of brain activity, the data can be embedded into a lower dimension Euclidean space using the concept of diffusion distances [11], as follows.

An fMRI volume at each time-point $t=1 \ldots T$ is treated as a vertex on a completely connected graph, specified by the $T \times T$ affinity matrix $\mathbf{W}$, where $\mathbf{W}_{t_{1}, t_{2}}=$ $\exp \left\{-E M D\left(S\left(t_{1}\right), S\left(t_{2}\right)\right)^{2} / 2 \sigma^{2}\right\}$. The user-defined parameter $\sigma$ defines a notion of proximity between activation patterns. Let $\mathbf{D}_{t, t}=\sum_{t^{\prime}=1}^{T} \mathbf{W}_{t, t^{\prime}}$ be the $T \times T$ diagonal degree matrix. Then $\mathbf{M}=\mathbf{D}^{-1} \mathbf{W}$ is a stochastic matrix (i.e. $\sum_{t^{\prime}=1}^{T} \mathbf{M}_{t, t^{\prime}}=1$ ) defining a random walk on the graph, with $\mathbf{M}_{t_{1}, t_{2}}$ as the probability $p\left(t_{2} \mid t_{1}\right)$ for a transition from node $S\left(t_{1}\right)$ to $S\left(t_{2}\right)$.

The probability $p\left(n, t_{2} \mid t_{1}\right)$ that the random walk starting at $S\left(t_{1}\right)$ will end at $S\left(t_{2}\right)$ in $n$-steps is given by $\mathbf{M}_{t_{1}, t_{2}}^{n}$. If the generalized eigen-system of $\mathbf{M}=\Psi \Lambda \Phi^{\prime}$, with $\Lambda$ the diagonal matrix of eigenvalues $1=\lambda_{1} \geq \ldots \geq \lambda_{T} \geq 0, \quad \Phi$ the matrix of right eigenvectors $\left(\phi_{1} \ldots \phi_{T}\right)$ and $\Psi$ that of left eigenvectors $\left(\psi_{1} \ldots \psi_{T}\right)$, then $p\left(n, t_{2} \mid t_{1}\right)=$ $\phi_{1}\left(t_{2}\right)+\sum_{j=2}^{T} \lambda_{j}^{n} \phi_{j}\left(t_{2}\right) \psi_{j}\left(t_{1}\right)$. Note that $\phi_{1}\left(t_{2}\right)$ is the stationary distribution $\lim _{n \rightarrow \infty} p\left(n, t_{2} \mid t_{1}\right)$ of the random walk, and is independent of starting point $S\left(t_{1}\right)$.

The diffusion distance between two vertices (i.e. fMRI scans) is defined as :

$$
D_{n}^{2}\left(S\left(t_{1}\right), S\left(t_{2}\right)\right)=\sum_{t=1}^{T}\left|p\left(n, t \mid t_{1}\right)-p\left(n, t \mid t_{2}\right)\right|^{2} \phi_{1}(t)=\sum_{j=1}^{T} \lambda_{j}^{2 n}\left(\psi_{j}\left(t_{2}\right)-\psi_{j}\left(t_{1}\right)\right)^{2} .
$$

The parameter $n$ defines the scale of the diffusion process and controls the sensitivity of the metric on the local geometry, with smaller $n$ making the metric more sensitive to local differences. It can be seen that $D_{n}^{2}\left(S\left(t_{1}\right), S\left(t_{2}\right)\right)$ is an Euclidean distance if the coordinates of $S(t)$ are given by $\left(\lambda_{j}^{n} \psi_{j}(t) \ldots \lambda_{T}^{n} \psi_{j}(t)\right)$.

The diffusion distance, though not equal to the geodesic distance, is related to the Laplace-Beltrami and Fokker-Planck operators on the manifold underlying the graph, and therefore provides a geometrically aware embedding for functions intrinsically defined on it [1]]. Moreover, since the spectral gap is usually large, with a few eigenvalues close to 1 and most $\approx 0$, the diffusion distance can be well-approximated by only the first $\hat{T} \ll T$ eigenvectors, with error of the order of $\mathcal{O}\left(\lambda_{\hat{T}+1}^{n}\right)$.

Hierarchical Clustering: After embedding the volumes in this $\hat{T}$-dimensional Euclidean space, they are grouped into $K$ clusters $\left\{c_{1} \ldots c_{K}\right\}$ using agglomerative hierarchical clustering. These clusters represent distinctive patterns of distribution of the BOLD signal and are probable indicators of the distinctive modes/states of cognition. Next, each cluster $c_{k}, k=1 \ldots K$ is labeled with an integer value $0 \leq l_{k}<K$, using dynamic programming to minimize $\sum_{t=1}^{T-1}\left|l_{k}(t+1)-l_{k}(t)\right|$, where $l_{k}(t)$ is the label of cluster $c_{k}$ if $S(t) \in c_{k}$. This results in a time series of labels where most transitions are between labels close to each other in value. 


\section{Results}

The method was applied on a data-set of 4 participants, who underwent fMRI while judging the incorrectness of multiplication results. In each trial, two single-digit numbers were either displayed visually or sounded out for $2.5 \mathrm{~s}$. After an interval of $0.3 \mathrm{~s}$ a solution was displayed or sounded for $0.8 \mathrm{~s}$. Subjects had up to $4 \mathrm{~s}$ to decide, with a button press, if the solution offered was either: (a) close (within $\pm 25 \%$ of the correct answer), (b) too small or (c) too big. The next trial started after a rest of $1 \mathrm{~s}$, and each trial ranged from $4 \mathrm{~s}$ to a maximum of $8.6 \mathrm{~s}$.

Acquisition was done on a GE 3T LX scanner with a quadrature head coil using a BOLD sensitized 2D-EPI gradient-echo pulse sequence $\left(\mathrm{TE}=35 \mathrm{~ms}, \mathrm{FA}=90^{\circ}, \mathrm{TR}=2 \mathrm{~s}\right.$, voxel size $3.75 \times 3.75 \times 3.75 \mathrm{~mm}^{3}$ ). A typical session lasted $\approx 18$ minutes, with 150 trials and 525 scans. The algorithms were implemented using MATLAB ${ }^{\circledR}$ and Star- $\mathrm{P}^{\circledR}$ on an $2.6 \mathrm{GHz}$ Opteron cluster with 16 processors and 32GB RAM.

The recursive EMD approximation was done with $J=10$ and thresholds $\tau_{j}$ were selected adaptively, so that only a small percentage $(25 \%)$ of the comparisons would need to be performed again at the next level. This resulted in a speed up of $10^{3} \times$, with an average running time of $\approx 23 \mathrm{hrs}$. per subject. Fig. 1 shows the relative approximation error $\left(E M D_{\text {true }}-E M D_{\text {approx }}\right) / E M D_{\text {true }}$ with respect to the true EMD. It is observed that the relative error scales linearly with respect to the true distance and is acceptably small for our data-sets.

The parameter $\sigma$ used in the affinity matrix W was set such that $\alpha \%$ of all the pair-wise EMDs were less than it, reflecting the assump-

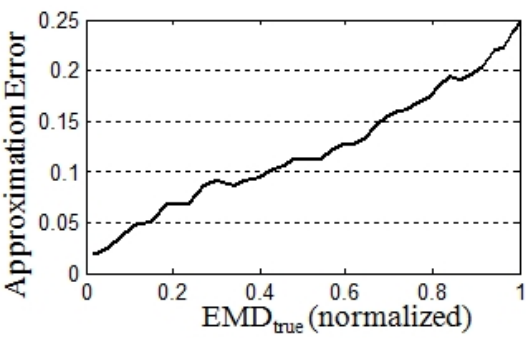

Fig. 1. Relative approximation error $\left(E M D_{\text {true }}-E M D_{\text {approx }}\right) / E M D_{\text {true }}$ with respect to $E M D_{\text {true }}$. The $x$-axis is normalized with respect to the maximum EMD. tion that $\sqrt{\alpha} \%$ of scans should be "close" to

any given scan. We found the results to be reproducible for $5 \% \leq \alpha \leq 20 \%$ in our experiments. For the low dimensional embedding in the diffusion metric space, $\hat{T}=8$ was a conservative value with $\lambda_{\hat{T}+1}<0.05$. The number of clusters $K=11$ was selected for all subjects.

In Fig. 2, the median brain-state labels for the audio and visual presentations of the trial are shown, for the four subjects. Here, a strong pattern in the assignment of the state with the phase of the task can be seen. Also, there is a clear separation of labels during presentation of the experiment depending on its modality (audio vs. visual), which converge towards the computation phase, as expected. These findings are especially significant given that no information about the experiment was used when determining the brain state. This synchronization of the brain-state labels with the experiment phase becomes more apparent on examining the intensity distributions for each cluster. The $t$-score maps for the first subject are shown in Fig. 3. The $t$-scores at every voxel were computed as within-cluster mean divided by within-cluster standard deviation. 


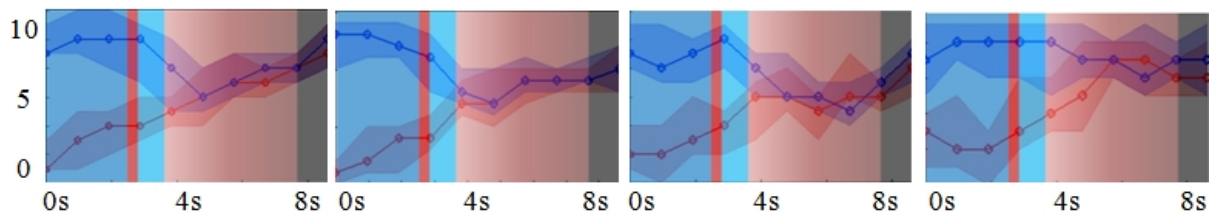

Fig. 2. The median brain-state labels, for all four subjects, during a single trial of the experiment. The phases of the experiment are color-coded to indicate the $2.5 \mathrm{~s}, 0.3 \mathrm{~s}, 0.8 \mathrm{~s}, 0-4 \mathrm{~s}$ and $1 \mathrm{~s}$ intervals of each trial. Red and blue lines show the the median brain-states for the visual vs. audio presentation of the numbers, respectively. Also shown are with the 25 and 75 percentile bands.

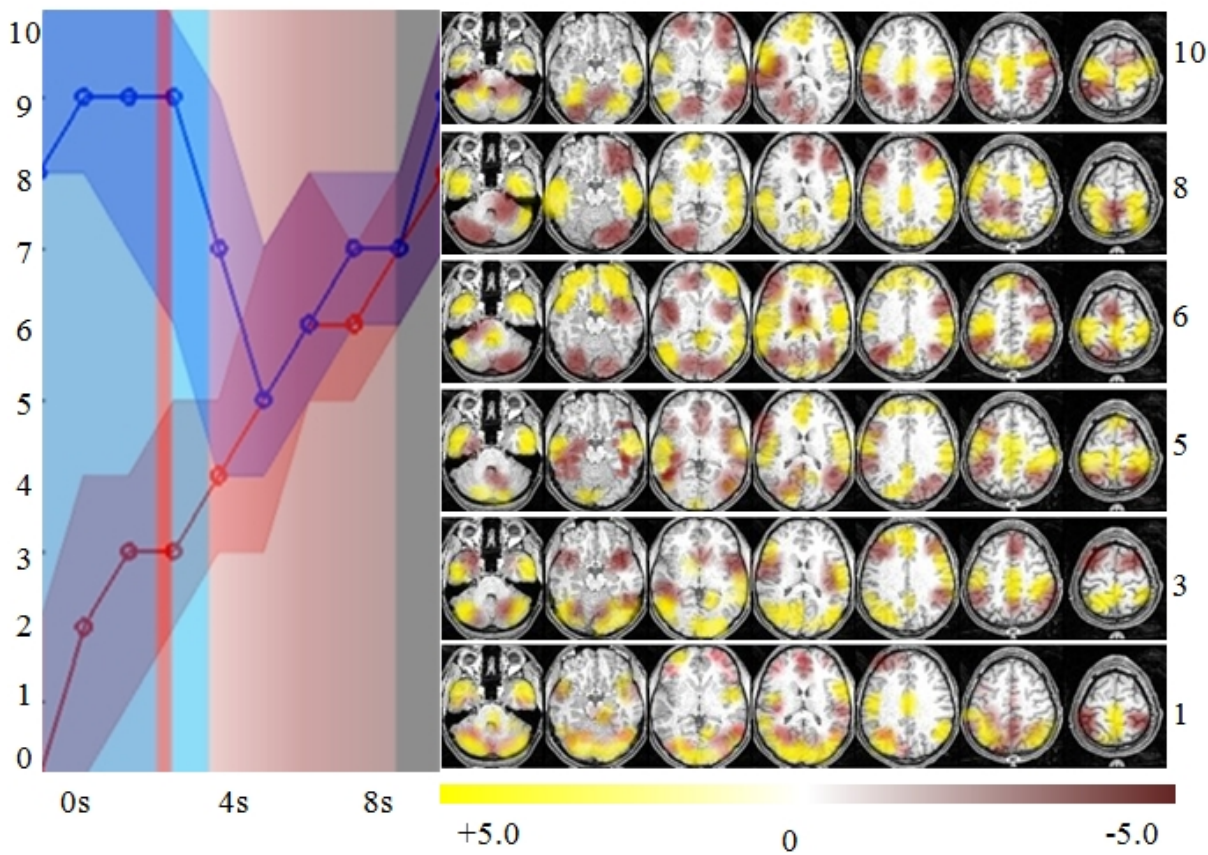

Fig. 3. The within-cluster $t$-scores for states $1,3,5,6,8,10$ for the first subject, overlaid on a highresolution structural scan. The color coding indicates the intensity in that region. Also shown is the temporal ordering of brain-states for the subject in each trial. The other maps are qualitatively similar and omitted for purposes of concision, while $t$-scores between $[-2,+2]$ are not displayed for clarity.

State 1 shows strong patterns in the visual cortex, and its occurrence typically corresponds to the visual presentation of the two numbers. State 3 , which usually occurs later, is active in visual areas related with size estimation. States 5 and 6 , associated with the calculation / judgement phase, are mainly in the frontal and parietal lobes, implicated in higher level cognition and number size assessment. There is also activity in the motor cortex and may be related to the button press. In states 8 and 10, which usually coincide 
with the audio presentation of the multiplication problem, the patterns in concentrated in the auditory cortices in the temporal lobe. These findings are in close agreement with those reported [12] for this paradigm using conventional analysis. While there are many unresolved issues in the interpretation of these maps, like negative values and statistical significance, their physiological consistency points to the validity of our method and affirms the potential of such approaches to analyze fMRI data.

\section{Conclusion}

In this paper, we presented a purely data-driven approach to learn the instantaneous state of the brain from the distribution of intensities in fMRI scans. We used a combination of the Earth Mover's Distance and diffusion distance to induce a metric on the space of fMRI volumes that was aware of its underlying geometry, and performed clustering in this space. We developed a computationally tractable approximation of the EMD based on recursive aggregation. Currently we are working on developing faster and better approximations to the EMD.

The method was applied on a study of arithmetical processing and a pattern in the sequence of brain states was observed that was highly organized with respect to the experiment, with distinct changes from one phase to another. Also the effect of different experimental conditions on the measured response of the brain was observed. Brain maps of activity were obtained that were physiologically meaningful with respect to the expected mental phase, and corresponded with the results of conventional analysis. Though there are many open computational and statistical issues, these results are remarkable given that they were derived solely from the fMRI data, and that no prior information about the experiment or subject behavior was used.

The combination use of EMD and diffusion distance is the main reason the method is able to extract relevant structures from the data. The diffusion distance could be thought of as an operator that uses locally accurate measures of similarity to induce a globally consistent Euclidean metric on the space. For it to succeed however, it is crucial that the underlying measure be accurate when two points are close to each other. In our experiments, we observed that a voxel-wise Euclidean distance (not reported here) performed much worse, as compared to the EMD, resulting in significantly less coherent clusters. This could be because the EMD is less sensitive to small but inevitable differences between signatures corresponding to the same conceptual brain state.

We are currently exploring methods to use these clusters to classify the brain-states of previously unseen scans of the same and of other subjects. Another avenue of research is to learn mappings between the brain states and observed behaviors, allowing for the potential of "brain reading". We are also interested in using information about experimental conditions to improve the identification of the brain-states, using semisupervised learning algorithms. We believe that such a method coupled with improvements in the temporal resolution of fMRI could give more insight into the temporal organization of the metabolic fingerprints of cognition. Also of interest is the problem of learning the simpler cognitive sub-states, that arise from parallel processes in the different functional circuits of the brain, but which combine in different ways to give rise to the more complex higher level cognition. 


\section{References}

[1] Haynes, J.D., Rees, G.: Decoding mental states from brain activity in humans. Nat, Rev. Neurosci. 7(7), 523-534 (2006)

[2] LaConte, S.M., Peltier, S.J., Hu, X.P.: Real-time fMRI using brain-state classification. Hum. Brain Mapp. 28(10), 1033-1044 (2007)

[3] Mitchell, T.M., Shinkareva, S.V., Carlson, A., Chang, K.M., Malave, V.L., Mason, R.A., Just, M.A.: Predicting Human Brain Activity Associated with the Meanings of Nouns. Science 320(5880), 1191-1195 (2008)

[4] Poldrack, R.A.: The role of fMRI in cognitive neuroscience: where do we stand? Curr. Opin. Neurobiol. 18(2), 223-227 (2008)

[5] Eklund, A., Ohlsson, H., Andersson, M., Rydell, J., Ynnerman, A., Knutsson, H.: Using real-time fMRI to control a dynamical system by brain activity classification. Med. Image Comput. Assist. Interv. 12(Pt 1), 1000-1008 (2009)

[6] Lehmann, D., Pascual-Marqui, R.D., Strik, W.K., Koenig, T.: Core networks for visualconcrete and abstract thought content: a brain electric microstate analysis. Neuroimage 49(1), 1073-1079 (2010)

[7] Rubner, Y., Tomasi, C., Guibas, L.J.: The earth mover's distance as a metric for image retrieval. Int. J. Comput. Vision 40(2), 99-121 (2000)

[8] Friston, K., Holmes, A., Worsley, K., Poline, J., Frith, C., Frackowiak, R.: Statistical parametric maps in functional imaging: A general linear approach. Hum. Brain Map. 2(4), 189-210 (1995)

[9] Alexander, M., Baumgartner, R., Windischberger, C., Moser, E., Somorjai, R.: Wavelet domain de-noising of time-courses in MR image sequences. Magn. Reson. Imaging 18(9), 1129-1134 (2000)

[10] Ng, B., Abugharbieh, R., Mckeown, M.J.: Functional segmentation of fMRI data using adaptive non-negative sparse PCA (ANSPCA), pp. 490-497 (2009)

[11] Coifman, R., Lafon, S.: Diffusion maps. Applied and Computational Harmonic Analysis, Special Issue on Diffusion Maps and Wavelets 21, 5-30 (2006)

[12] Morocz, I., Gross-Tsur, A., von Aster, M., Manor, O., Breznitz, Z., Karni, A., Shalev, R.: Functional magnetic resonance imaging in dyscalculia: preliminary observations. Annals of Neurology 54(S7), S145 (2003) 